\title{
International Conference on
}

\section{Medical Parasitology and Zoology \\ October 17-19, 2016 Houston, USA}

\section{Does hydatid cyst fluid from Echinococcus granulosus cysts have any effect on cells involved in fibrosis in cystic echinococcosis?}

Ahmed Ali Mohammed, Allen Jeremy and Rogan Michael T

University of Salford, UK

\begin{abstract}
Cystic echinococcosis is characterized by the presence of slow growing hydatid cysts, usually in the liver or lungs. Survival of the $\checkmark$ parasite is based on an interaction of the host immune system and a range of parasite immune-evasive strategies. Fibrosis in the tissues surrounding the cysts can be seen as a host protective response isolating the parasite and restricting its growth or from another perspective fibrosis may be protective for the parasite by providing a barrier to more effective immunological responses. In this study the adenocarcinomic human alveolar basal epithelial cell line (A549) was used as model system. This cell line can be involved in fibrosis as cells can transform into mesenchymal cells and differentiate later to fibroblasts and/or myofibroblasts which can ultimately secrete collagen. Cells were initially cultured in vitro in RPMI-1640 medium containing 1-10\% hydatid cyst fluid (HCF). The possible effect of the parasite extracts on cell migration was investigated using a wound healing assay. The ability of HCF components to modify cell surface markers of mesenchymal transition was also investigated by fluorescence microscopy. Results showed that there was a dose-dependent increase in cell growth in the presence of cyst fluid after 5 days of culture. The migratory response of cells was also enhanced by the presence of HCF. Both the enhanced growth and migratory activity were still evident when the HCF had been boiled indicating that the components responsible were thermostable. Semi-purified extracts of a major HCF component, antigen B showed a similar high stimulatory effect similar to that of HCF. The fluorescence microscopy showed a significant expression in the fibronectin and E-cadherin cell markers in cells treated with HCF. These results indicate that components within HCF have a stimulatory effect in the possible enhancement of fibrosis.
\end{abstract}

\section{Biography}

Ahmed Ali Mohammed has completed his MSc in Immunoparasitology from the University of Baghdad, College of Science and currently pursuing his PhD in the University of Salford, School of Environment and Life Sciences-Biomedical Research Centre. He has been working as a formal Staff Member (Assistant Professor) in the Branch of Clinical Laboratory Sciences in the College of Pharmacy Al-Mustansiriyah University in Iraq. He was granted a patent in 2002 and has 11 published papers in local Iraqi reputed journals. He has authored two curricular books and one practical guide booklet in Medical Parasitology for his students of pharmacy. He has also served as a Referee in three valued journals one in Iraq and two international journals. 